\title{
Estimating black hole masses of blazars
}

\author{
Xue-Bing Wu*1, F. K. Liu ${ }^{1}$, M. Z. Kong ${ }^{2}$, R. Wang ${ }^{3}$, J. L. Han ${ }^{4}$ \\ ${ }^{1}$ Department of Astronomy, Peking University, Beijing 100871, China \\ ${ }^{2}$ Department of Physics, Hebei Normal University, Shijiazhang 050016, China \\ ${ }^{3}$ Steward Observatory, University of Arizona, Tucson, AZ 85721, USA \\ ${ }^{4}$ National Astronomical Observatories, Chinese Academy of Sciences, Beijing 100012, China
}

2018 November 7

\begin{abstract}
Estimating black hole masses of blazars is still a big challenge. Because of the contamination of jets, using the previously suggested size - continuum luminosity relation can overestimate the broad line region (BLR) size and black hole mass for radio-loud AGNs, including blazars. We propose a new relation between the BLR size and $H_{\beta}$ emission line luminosity and present evidences for using it to get more accurate black hole masses of radio-loud AGNs. For extremely radio-loud AGNs such as blazars with weak/absent emission lines, we suggest to use the fundamental plane relation of their elliptical host galaxies to estimate the central velocity dispersions and black hole masses, if their velocity dispersions are not known but the host galaxies can be mapped. The black hole masses of some well-known blazars, such as OJ 287, AO 0235+164 and 3C 66B, are obtained using these two methods and the $\mathrm{M}-\sigma$ relation. The implications of their black hole masses on other related studies are also discussed.
\end{abstract}

Key words: Black holes - galaxies:active - blazars - jets

\section{Introduction}

In the last two decades, dynamical measurements have clearly revealed that supermassive black holes exist in the center of nearby galaxies. However, dynamical methods can not be applied to most of AGNs because they are too bright. Currently the most reliable method for AGN black hole mass estimation is the reverberation mapping. Using this technique, the BLR size can be measured using the time lag between the variations of continuum and emission line fluxes. The black hole mass can be derived from the BLR size and the characteristic velocity using the virial law. So far, reverberation mapping studies have yielded black hole masses of about

\footnotetext{
*e-mail:wuxb@pku.edu.cn
} 
40 Seyfert 1 galaxies and nearby quasars ( Kaspi et al. 2000, 2005; Peterson et al. 2004). Using the observed data of these reverberation mapping AGNs, an empirical relation between the BLR size $(R)$ and continuum luminosity at $5100 \AA$ $\left(L_{5100}\right)$ has been derived by Kaspi et al. $(2000,2005)$, which has been frequently adopted to estimate the BLR size and the black hole masses for large samples of AGNs, including radio-loud quasars. However, the optical continuum luminosity of radio-loud AGNs may not be a good indicator of ionizing luminosity. Powerful jets of blazar-type AGNs may significantly contribute to the optical continuum luminosity. Therefore, using the $R-L_{5100 \AA}$ relation may significantly overestimate the actual BLR size and the black hole mass of radio-loud AGNs. In addition, another tight correlation between black hole mass and bulge velocity dispersion $(\sigma)$ has been found for nearby galaxies (Tremaine et al. 2002) and for a few Seyfert galaxies as well (Ferrarese et al. 2001). Such a relation suggests a possibility to estimate the black hole masses of AGNs using the measured bulge velocity dispersions. Especially for BL Lacertae objects, the reverberation mapping technique cannot be applied because they show no or weak emission lines in their optical spectra. Using the $\mathrm{M}_{B H^{-}}-\sigma$ relation may be the only way to derive their black hole masses, though measuring $\sigma$ is possible only for nearby sources. We have to seek for other methods for most blazars because the velocity dispersions of their host galaxies are too difficult to be measured.

In this paper we report our progress on estimating the black hole masses of radio-loud AGNs using a new BLR size $-H_{\beta}$ emission line luminosity relation and the fundamental plane relation of the elliptical host galaxies. The results on some well-known blazars are also presented.

\section{The BLR size $-\mathbf{H}_{\beta}$ luminosity relation}

Using the available data of BLR sizes and $\mathrm{H}_{\beta}$ fluxes for 34 AGNs in the reverberation mapping studies (Kaspi et al. 2000), we investigated the relation between the BLR size and the $\mathrm{H}_{\beta}$ emission line luminosity ( $\mathrm{Wu}$ et al. 2004). An empirical relation between the BLR size and $\mathrm{H}_{\beta}$ luminosity was derived as:

$\log R(l t-$ days $)=(1.381 \pm 0.080)+(0.684 \pm 0.106) \log \left(\mathrm{L}_{\mathrm{H}_{\beta}} / 10^{42} \operatorname{ergs~s}^{-1}\right)$.

The Spearman's rank correlation coefficient of this relation is 0.91 . In Fig. 1 we show the dependence of the BLR size on $L_{H_{\beta}}$ and $L_{5100 \AA}$. Obviously these two relations are similar, which means that the $R-L_{H_{\beta}}$ relation can be an alternative of the $R-L_{5100 \AA}$ relation in estimating the BLR size for radio-quiet AGNs. We applied both the $R-L_{H_{\beta}}$ and $R-L_{5100 \AA}$ relations to estimate the black hole masses of 87 radio-loud quasars and compare them in Fig. 2. Evidently the masses obtained with the $R-L_{H_{\beta}}$ relation are systematically lower that those obtained with the $R-L_{5100 \AA}$ relation for some extremely radio-loud quasars. The difference between two black hole mass estimates is smaller when the radio-loudness is small but becomes larger as the radio-loudness increases. For some individual quasars with 
higher radio-loudness, the black hole mass estimated with the $R-L_{5100 \AA}$ relation can be 3 10 times larger than that estimated with the $R-L_{H_{\beta}}$ relation. Recently Kong et al. (2006) also extended such a study to the broad UV emission lines MgII and CIV, and obtained the BLR size - UV emission line luminosity relations. Our results demonstrated that using the BLR size - emission line luminosity relations can avoid the overestimations of the black hole masses for blazar-like radio-loud AGNs.
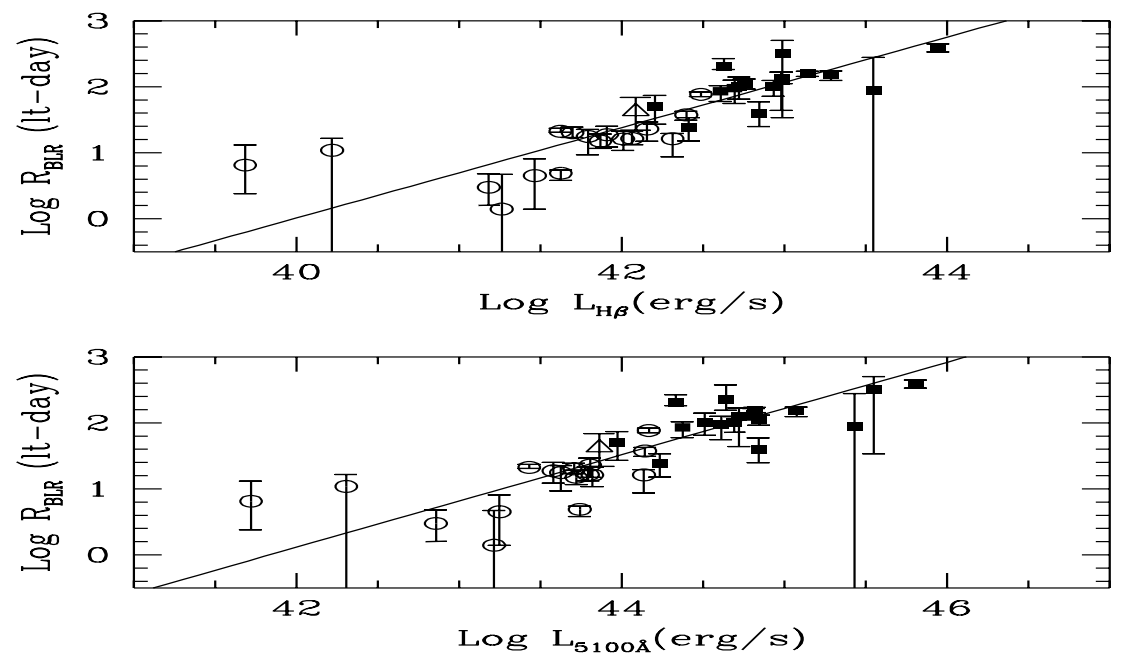

Figure 1. The $R-L_{H_{\beta}}$ relation and the $R-L_{5100 \AA}$ relation. The open and filled symbols denote Seyferts and quasars respectively. The figure is taken from Wu et al. (2004).

\section{Black hole masses estimated from the fundamental plane relation of AGN elliptical host galaxies}

For radio-loud AGNs such as BL Lacs with weak/absent emission lines, the emission line based methods for black hole mass estimations can not apply. Directly measuring their stellar velocity dispersion is also difficult. However, the host galaxies of BL Lacs are virtually ellipticals. It is well known for ellipticals that three observables, namely the effective radius $\left(R_{e}\right)$, the average surface brightness $\left(<\mu_{e}>_{R}\right.$ in R-band) and the central velocity dispersion $(\sigma)$, follow a tight fundamental plane relation. For about 300 normal ellipticals and radio galaxies, Bettoni et al. (2001) found that the fundamental plane can be robustly described as

$$
\log R_{e}=(1.27 \pm 0.04) \log \sigma+(0.326 \pm 0.007)<\mu_{e}>_{R}-8.56 \pm 0.06,
$$

This relation provides us another way to estimate the central velocity dispersions and then the black hole masses of AGNs (Wu, Liu \& Zhang 2002). 

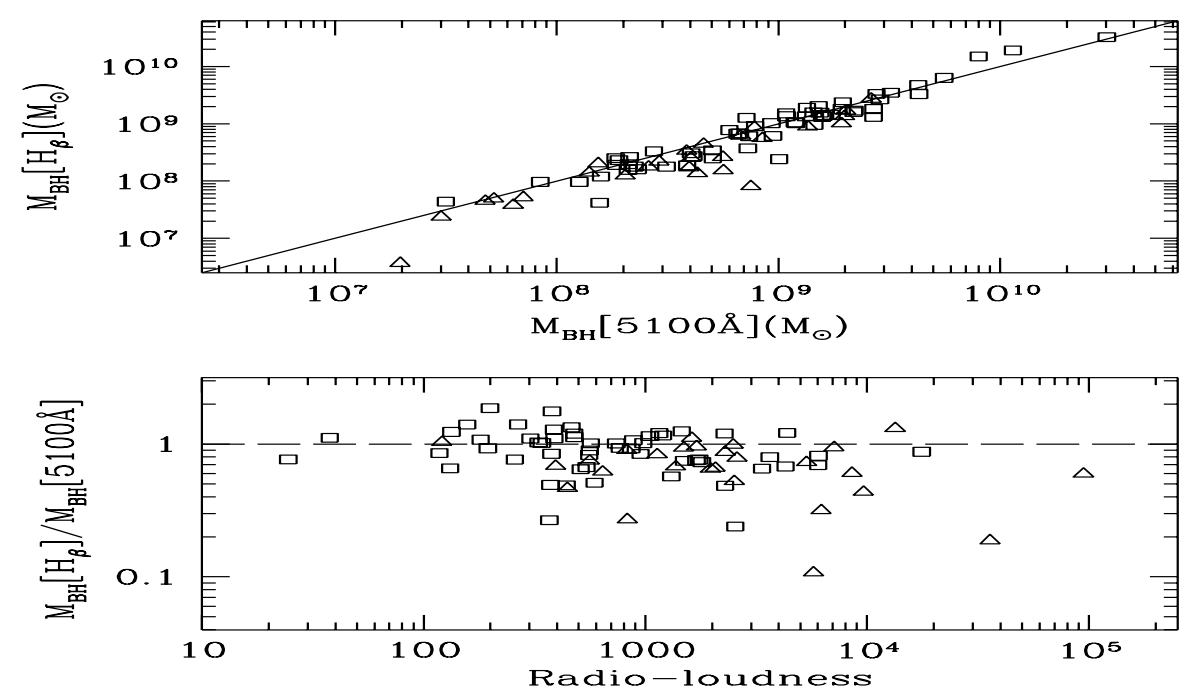

Figure 2. Comparison of the black hole masses of radio-loud quasars estimated with two $\mathrm{R}-\mathrm{L}$ relations, and the dependence of the black hole mass difference on radio loudness. The figure is taken from $\mathrm{Wu}$ et al. (2004).

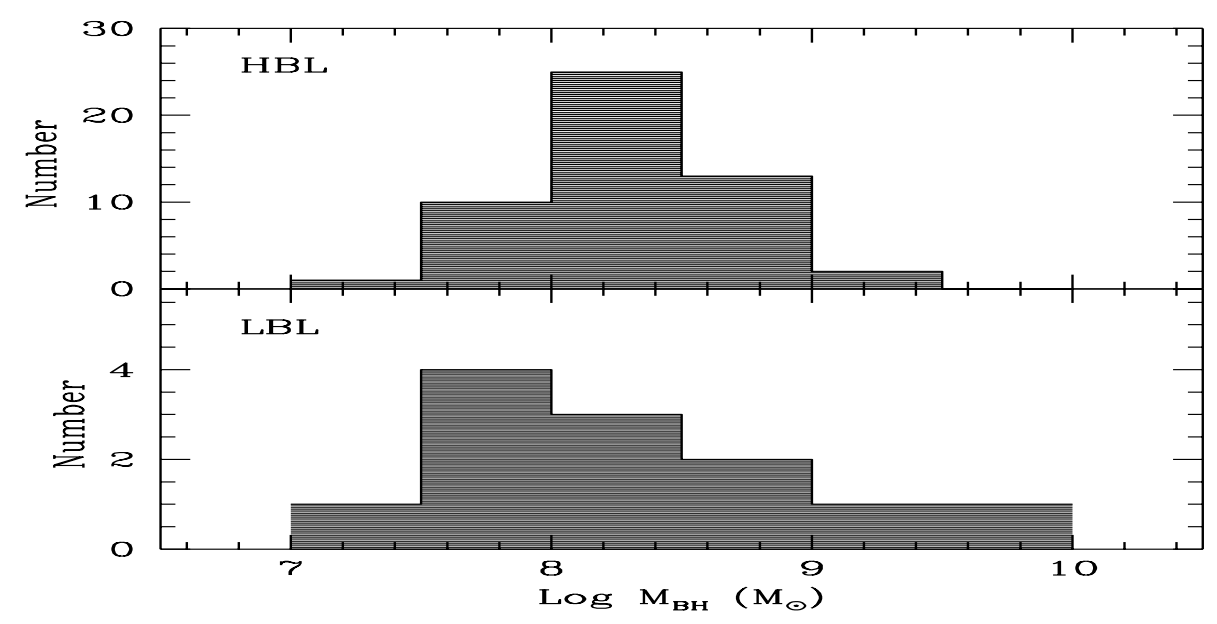

Figure 3. Histograms of the derived black hole mass distribution of HBLs and LBLs. The figure is taken from $\mathrm{Wu}$ et al. (2002).

Using the imaging data of BL Lacs obtained from the HST snapshot survey (Urry et al. 2000), we adopted the fundamental plane relation to estimate the central velocity dispersions and black hole masses of 51 high-frequency peaked BL Lacs (HBLs) and 12 low-frequency peaked BL Lacs (LBLs). Our results show no significant difference in the black hole masses between HBLs and LBLs (see Fig. 3). We also applied the same method to 10 radio galaxies (RGs), 10 radio- 


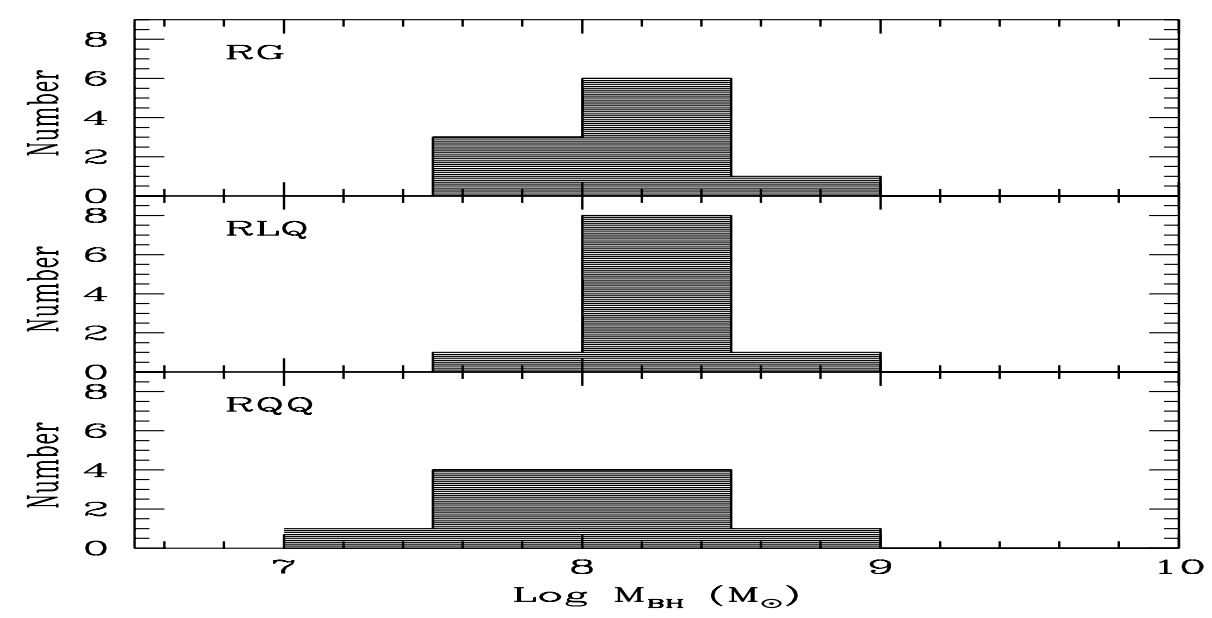

Figure 4. Histograms of the derived black hole mass distribution of radio galaxies, radioloud and radio-quiet quasars. The figure is taken from $\mathrm{Wu}$ et al. (2002).

loud quasars (RLQs) and 13 radio-quiet quasars (RQQs) which have been imaged by HST (McLure et al. 1999), we found that there are no significant differences in the black hole masses among these different types of AGNs with elliptical host galaxies (see Fig. 4).

\section{Black hole masses of three well-known blazars}

\subsection{AO $0235+164$}

Liu, Zhao, \& Wu (2006) applied the $R-L_{H_{\beta}}$ relation suggested by Wu et al. (2004) to a well studied BL Lac object AO $0235+164$ and estimated its black hole mass as $5.8 \times 10^{8} M_{\odot}$, which is consistent with the mass $\left(3.6 \times 10^{8} M_{\odot}\right)$ estimated from the $M_{B H}-\sigma$ relation by taking the narrow emission line width as a surrogate of $\sigma$, but is much smaller than the mass $\left(1.5 \times 10^{9} M_{\odot}\right)$ obtained from the $R-L_{5100 \AA}$ relation. This again demonstrates that using the previous $R-L_{5100 \AA}$ relation can overestimate the black hole masses of blazar-like AGNs. Our study indicates that the black hole mass of $\mathrm{AO} 0235+164$ is most likely around $5 \times 10^{8} M_{\odot}$.

\subsection{OJ 287}

We applied the fundamental plane relation of ellipticals to a well-known BL Lac object OJ 287, which is a possible supermassive black hole binary system. Liu \& $\mathrm{Wu}(2002)$ estimated its primary black hole mass to be about $4 \times 10^{8} M_{\odot}$, which is consistent with the upper limit $\left(10^{9} M_{\odot}\right)$ obtained by Valtaoja et al. (2000) based on a binary black hole model for OJ 287. This value is also within the range of typical black hole masses for BL lac objects (Wu, Liu \& Zhang 2002). We noticed 
that our derived black hole mass is much smaller than $1.8 \times 10^{10} M_{\odot}$, which is required in a new precessing binary black hole model of OJ 287 (Valtonen 2007).

\section{$4.33 C 66 B$}

3C 66B is a well-known nearby FR I radio galaxy. The central velocity dispersion of 3C 66B has been obtained by Balcells et al. (1995) as $348 \pm 29 \mathrm{~km} / \mathrm{s}$ based on their spectroscopic observations. Using the $\mathrm{M}-\sigma$ relation suggested by Tremaine et al. (2002), we estimate that $\log \left(M / M_{\odot}\right)=9.097 \pm 0.175$, namely the mass of the central black hole of $3 \mathrm{C} 66 \mathrm{~B}$ is about $1.25 \times 10^{9} M_{\odot}$. This is within the range of typical black hole masses for radio galaxies (Wu, Liu \& Zhang 2002), but one-order of magnitude smaller than the maximum mass estimated by Sudou et al. (2003), $5 \times 10^{10} M_{\odot}$, which was obtained by modeling the observed orbital motion with a binary black hole model. A very recent study by Iguchi et al. (2010) supported that the larger black hole mass is $\left(1.2_{-0.2}^{+0.5}\right) \times 10^{9} M_{\odot}$, which is consistent with the value obtained by the $\mathrm{M}$ - $\sigma$ relation. Janet et al. (2004) adopted the black hole mass of 3C 66B obtained by Sudou et al. (2003) to calculate the gravitational wave emission from $3 \mathrm{C}$ 66B and argued that the binary black hole model can be excluded because observations from the Pulsar Timing Array (PTA) did not detect the gravitational wave from $3 \mathrm{C}$ 66B. However, if the smaller black hole mass is adpted, the gravitational wave emitted from $3 \mathrm{C} 66 \mathrm{~B}$ will be weaker than the current PTA detection limit and the binary black hole model can not be excluded.

\section{Summary}

We proposed to use the BLR size - emission line luminosity relation and the fundamental plane relation of the elliptical host galaxies to estimate the black hole masses of radio-loud AGNs. We demonstrated that with the first relation we can get more accurate black hole mass estimates for radio-loud AGNs than using the usual $R-L_{5100 \AA}$ relation, and for some radio-loud AGNs such as BL Lacs the second method is probably the only available one for their black hole mass estimations in the case when directly measuring the stellar velocity dispersions is difficult. We have adopted these methods, as well as the $\mathrm{M}-\sigma$ relation, to estimate the black hole masses of three well-known blazars, AO 0235+164, OJ 297 and 3C 66B, and the results are helpful to other related study about their physics nature. Finally, we would like to mention that these two methods can be also applied to estimate the black hole masses of high redshift AGNs, including blazars, with high quality spectroscopic and imaging observations.

This work is supported by the NSFC Grants (No. 10473001, 10573001, 10525313 and 11033001) and the 973 program (No. 2007CB815405).

\section{References}

Balcells, M. et al. 1995, A\&A, 302, 665 
Bettoni, D. et al. 2001, A\&A, 380, 471

Ferrarese, L. et al. 2001, ApJ, 555, L79

Iguchi, S., Takeshi, O., \& Sudou, H. 2010, ApJ, 724, L166

Jenet, F.A., et al. 2004, ApJ, 606, 799

Kaspi, S. et al. 2000, ApJ, 533, 631

Kaspi, S. et al. 2005, ApJ, 629, 61

Kong, M. Z., Wu, X.-B., Wang, R., Han, J. L. 2006, Chin. J. Astron. Astrophys. 6, 396

Liu, F. K., \& Wu, X.-B., 2002, A\&A, 388, L48

Liu, F. K., Zhao, G., \& Wu, X.-B., 2006, ApJ, 650, 749

McLure, R. J., et al. 1999, MNRAS, 308, 377

Peterson, B. M. et al. 2004, ApJ, 613, 682

Sudou, H. et al., et al. 2003, Science, 300, 1263

Tremaine, S. et al. 2002, ApJ, 574, 740

Urry, C. M. et al. 2000, ApJ, 532, 816

Valtaoja, E. et al. . 2000, ApJ, 531, 744

Valtonen, M.J., 2007, ApJ, 659, 1074

Wu, X.-B., Liu, F. K., Zhang, T. Z. 2002, A\&A, 389, 742

Wu, X.-B., Wang, R., Kong, M. Z., Liu, F. K., Han, J. L., 2004, A\&A, 424, 793 